\title{
THE MINIMUM MISS DISTANCE PROBLEM ${ }^{1}$
}

\author{
T. F. BRIDGLAND, JR. ${ }^{2}$ AND J. S. HINKEL ${ }^{3}$
}

I. In the statement of most extant theorems concerning optimal control, a fundamental hypothesis is that of the existence of a control function which drives the controlled system from a given initial state to a specified target. Thus the utility of these theorems is contingent upon the solution of what is itself a fundamental and difficult problem of the mathematical theory of control: the so-called controllability problem. One thus is led to ask if it is possible, through suitable reformulation of the basic problem of optimal control, to prove analogues of existing theorems with a weaker hypothesis than that of controllability. That it may be possible in some cases is suggested by the comments in [1] concerning the minimum miss distance problem.

Stated loosely, the minimum miss distance problem is that of controlling the motion of an object (interceptor) in such a way as to minimize its distance of closest approach (miss distance) to another moving object (target). In [1], a mathematical formulation of this problem was presented for the case in which the interceptor is described mathematically by a system of ordinary differential equations involving a number of more or less arbitrary (control) functions and the target is described in mathematical generality sufficient to include most cases of interest in applications of optimal control theory.

In this paper we present a detailed analysis of the minimum miss distance problem as formulated in [1]. We show that: (i) if there is a control which moves the interceptor closer to the target (a weakening of the controllability hypothesis) then the conditions of Filippov's existence theorem [2] are sufficient to ensure the existence of a control which not only minimizes miss distance but does so in minimum time; (ii) among other topological properties, the set $B_{\infty}$ of initial data from which it is possible for the interceptor to get closer to the target possesses the property of being open (a property of substantial utility in the solution of optimal feedback control problems). Finally, we establish a simple criterion, in the case of a fixed point target, for the nonemptiness of the set $B_{\infty}$, the nonemptiness of this set being equivalent

Received by the editors June 6, 1966.

1 The research reported in this paper was subsidized by the National Aeronautics and Space Administration under research grants NGR 41-002-001 and NsG-381.

2 University of Alabama Research Institute, Huntsville.

- University of South Carolina. 
to the existence of initial data for which the weakened controllability hypothesis of (i) is satisfied.

II. Let $U$ denote a (control) set of vector-valued functions $u$ having bounded, Lebesgue measurable components and which map the set $I_{T}=\{t \mid 0 \leqq t \leqq T\}$ in to euclidean $m$-space $R^{m}$. Let $f$ be a continuous map of $I_{T} \times R^{n} \times R^{m}$; then for each $\left(t_{0}, x_{0}, u\right) \in I_{T} \times R^{n} \times U$, the local existence of a solution (in the sense of Carathéodory) of the differential equation

$$
\dot{x}=f(t, x, u(t)), \quad x\left(t_{0}\right)=x_{0},
$$

is assured. We assume that the solutions of (1) are unique and we denote the value of a solution by $x_{u}\left(t ; t_{0}, x_{0}\right)$. We assume further that for each $\left(t_{0}, x_{0}, u\right) \in I_{T} \times R^{n} \times U$, the solution may be continued over the interval $\left[t_{0}, T\right]$.

Let us denote by $\left\{\Omega^{n} ; d\right\}$ the space of all nonvoid, compact subsets of $R^{n}$, metrized by the Hausdorff distance, $d$. A continuous map $t \sim G(t)$ of $I_{T}$ into $\left\{\Omega^{n} ; d\right\}$ will be called a target $[1$, p. 264]. For a point $x \in R^{n}$ and a nonvoid compact subset $A \subset R^{n}$, the distance $\partial(x, A)$ between $x$ and $A$ is defined by

$$
\partial(x, A)=\min _{a \in \boldsymbol{A}}\|x-a\|
$$

where $\|\cdot\|$ denotes the norm in $R^{n}$. In $[1$, Lemma 1$]$ it is shown that the map $(x, A) \sim \partial(x, A)$ is continuous on $R^{n} \times\left\{\Omega^{n} ; d\right\}$.

In this paper we shall assume a prescribed target; we define a miss distance, $\delta(t, x, u)$, for (1) by

$$
\delta(t, x, u)=\min _{\tau \in[t, T]} \partial\left(x_{u}(\tau ; t, x), G(\tau)\right) .
$$

As shown in [1], the set

$$
\Im_{u}(t, x)=\left\{\tau \in[t, T] \mid \partial\left(x_{u}(\tau ; t, x), G(\tau)\right)=\delta(t, x, u)\right\}
$$

has a least element-the first time of closest approach-which we denote by $t^{*}(t, x, u)$. We note that the domain of definition of both $\delta$ and $t^{*}$ is $I_{T} \times R^{n} \times U$.

The set of admissible controls $U(t, x)$ is defined by

$$
U(t, x)=\left\{u \in U \mid t<t^{*}(t, x, u) \leqq T\right\}
$$

and the kernel, $K(t, x)$, of $U(t, x)$ is defined by

$$
K(t, x)=\left\{u \in U(t, x) \mid\left(t^{*}(t, x, u), x_{u}\left(t^{*}(t, x, u) ; t, x\right)\right) \notin B_{\infty}\right\}
$$

where 


$$
B_{\infty}=\left\{(t, x) \in I_{T} \times R^{n} \mid U(t, x) \text { is nonvoid }\right\} .
$$

Associated with $B_{\infty}$ are the following sets:

$$
\begin{aligned}
& B_{\gamma}=\left\{(t, x) \in B_{\infty} \mid \delta(t, x, u)<\gamma \text { for some } u \in U(t, x)\right\}, \quad 0<\gamma<\infty ; \\
& B_{0}=\bigcap_{0<\gamma} B_{\gamma} .
\end{aligned}
$$

Evidently $B_{\infty}=\mathrm{U}_{0<\gamma} B_{\gamma}$, so that $B_{\infty}$ is nonvoid if and only if $B_{\gamma}$ is nonvoid for some $\gamma \in(0, \infty)$.

Lemma 1. For each $T<\infty$ and each $u \in U, \delta$ is continuous on $I_{T} \times R^{n}$.

Proof. Setting

$$
\begin{aligned}
\phi(t) & =\min _{\tau \in[t, T]} \partial\left(x_{u}\left(\tau ; t_{0}, x_{0}\right), G(\tau)\right)-\min _{\tau \in\left[t_{0}, T\right]} \partial\left(x_{u}\left(\tau ; t_{0}, x_{0}\right), G(\tau)\right), \\
\psi(\tau, t, x) & =\partial\left(x_{u}(\tau ; t, x), G(\tau)\right)-\partial\left(x_{u}\left(\tau ; t_{0}, x_{0}\right), G(\tau)\right),
\end{aligned}
$$

the following estimate is an elementary consequence of the definition of $\delta$ :

$$
\begin{aligned}
\phi(t)+\min _{\tau \in[t, T]} \psi(\tau, t, x) & \leqq \delta(t, x, u)-\delta\left(t_{0}, x_{0}, u\right) \\
& \leqq \phi(t)+\max _{\tau \in[t, T]} \psi(\tau, t, x) .
\end{aligned}
$$

As a consequence of our assumptions concerning (1) and of [3, Theorem 4.3 , p. 59], it follows that when $t_{0} \in(0, T]$ there exist $\alpha_{1}>0$, $\alpha_{2}>0$ such that for $\left|t-t_{0}\right|<\alpha_{1},\left\|x-x_{0}\right\|<\alpha_{1}, \psi(\tau, t, x)$ is defined for all $\tau \in\left[t_{0}-\alpha_{2}, T\right]$. If $t_{0}=0$ the same assertion is true with $\left[t_{0}-\alpha_{2}, T\right]$ replaced by $I_{T}$. With this established, (2) may be replaced by

$$
\begin{aligned}
\phi(t)+\min \psi(\tau, t, x) & \leqq \delta(t, x, u)-\delta\left(t_{0}, x_{0}, u\right) \\
& \leqq \phi(t)+\max \psi(\tau, t, x)
\end{aligned}
$$

where the extremizations are on $\left[t_{0}-\alpha_{2}, T\right]$ when $t_{0}>0$ and on $I_{T}$ when $t_{0}=0$. In either case, the same theorem [3, loc. cit.], together with the continuity of $\partial, G$, implies the equicontinuity at $\left(t_{0}, x_{0}\right)$ of the family $(t, x) \sim \psi(\tau, t, x), \tau \in\left[t_{0}-\alpha_{2}, T\right]$ (or $\tau \in I_{T}$ if $t_{0}=0$ ). This equicontinuity and the continuity of $\phi$ at $t_{0}$ imply, by virtue of (3), the continuity of $\delta$ at $\left(t_{0}, x_{0}\right)$.

THEOREM 1. For every $\gamma, 0<\gamma<\infty, B_{\gamma}$ is relatively open in $I_{T} \times R^{n}$.

Proof. The assertion is trivial if $B_{\gamma}$ is empty. If $B_{\infty}$ is not empty then for $(t, x) \in B_{\infty}$ we define

$$
\rho(t, x, u)=\partial(x, G(t))-\delta(t, x, u)
$$

and note that $\rho(t, x, u)>0$ for $u \in U(t, x)$. For all $(t, x) \in I_{T} \times R^{n}$ such 
that $t \in\left[0, t^{*}\left(t_{0}, x_{0}, u\right)\right),\left(t_{0}, x_{0}\right) \in B_{\infty}$, we find

$$
\begin{aligned}
\partial(x, G(t)) & -\partial\left(x_{u}\left(t^{*}\left(t_{0}, x_{0}, u\right) ; t, x\right), G\left(t^{*}\left(t_{0}, x_{0}, u\right)\right)\right) \\
= & \rho\left(t_{0}, x_{0}, u\right)+\left[\partial(x, G(t))-\partial\left(x_{0}, G\left(t_{0}\right)\right)\right] \\
& +\left[\partial\left(x_{u}\left(t^{*}\left(t_{0}, x_{0}, u\right) ; t_{0}, x_{0}\right), G\left(t^{*}\left(t_{0}, x_{0}, u\right)\right)\right)\right. \\
& \left.\quad-\partial\left(x_{u}\left(t^{*}\left(t_{0}, x_{0}, u\right) ; t, x\right), G\left(t^{*}\left(t_{0}, x_{0}, u\right)\right)\right)\right] .
\end{aligned}
$$

It is then a consequence of (4), the continuity of $\partial$ and $G$ and the continuity with respect to initial parameters of the solutions of (1) that for $\epsilon>0$ there exists $\alpha=\alpha\left(t_{0}, x_{0}, u, \epsilon\right)>0$ such that if $\left|t-t_{0}\right|<\alpha$, $\left\|x-x_{0}\right\|<\alpha$ then

$$
\partial(x, G(t))-\partial\left(x_{u}\left(t^{*}\left(t_{0}, x_{0}, u\right) ; t, x\right), G\left(t^{*}\left(t_{0}, x_{0}, u\right)\right)\right)>\rho\left(t_{0}, x_{0}, u\right)-\epsilon .
$$

By taking $\epsilon \leqq \rho\left(t_{0}, x_{0}, u\right)$, this shows that $B_{\infty}$ is relatively open in $I_{T} \times R^{n}$. If $\left(t_{0}, x_{0}\right) \in B_{\gamma}, 0<\gamma<\infty$, then since for fixed $u$ and all $\left|t-t_{0}\right|$ $<\alpha,\left\|x-x_{0}\right\|<\alpha, u \in U\left(t_{0}, x_{0}\right) \cap U(t, x)$, the continuity of $\delta$ implies that, by taking $\left|t-t_{0}\right|,\left\|x-x_{0}\right\|$ still smaller if necessary, $(t, x) \in B_{\gamma}$. Hence, $B_{\gamma}$ is relatively open in $I_{T} \times R^{n}$.

Theorem 2. If $B_{\infty}$ is nonvoid and if there exists $C>0$ such that

$$
x \cdot f(t, x, u(t)) \geqq-C\left(\|x\|^{2}+1\right)
$$

for each $(t, x) \in B_{\infty}$ and all $u \in U(t, x)$ then for any nonvoid $B_{\gamma}, 0 \leqq \gamma$ $<\infty, B_{\gamma}$ is bounded.

Proof. From (1) and $\left({ }^{*}\right)$ there is obtained by integration the estimate

$$
\begin{gathered}
\left\|x_{0}\right\|^{2}+1 \leqq \\
\cdot\left(\left\|x_{u}\left(t^{*}\left(t_{0}, x_{0}, u\right) ; t_{0}, x_{0}\right)\right\|^{2}+1\right) \\
\cdot \exp 2 C\left(t^{*}\left(t_{0}, x_{0}, u\right)-t_{0}\right)
\end{gathered}
$$

for $\left(t_{0}, x_{0}\right) \in B_{\infty}, u \in U\left(t_{0}, x_{0}\right)$, since $\left(t, x_{u}\left(t ; t_{0}, x_{0}\right)\right) \in B_{\infty}$ for all $t \in\left[t_{0}\right.$, $\left.t^{*}\left(t_{0}, x_{0}, u\right)\right)$ and since $u \in U\left(t_{0}, x_{0}\right)$ implies $u \in U\left(t, x_{u}\left(t ; t_{0}, x_{0}\right)\right)$ on the same interval. From the definition of $\delta$ we find

$$
\gamma>\delta\left(t_{0}, x_{0}, u\right)=\left\|x_{u}\left(t^{*}\left(t_{0}, x_{0}, u\right) ; t_{0}, x_{0}\right)-\xi\right\|
$$

for some point $\xi \in G\left(t^{*}\left(t_{0}, x_{0}, u\right)\right)$. It is easy to show by means of a proof similar to that of $[1$, Lemma 1] that the function $A \sim$ $\max _{x \in A}\|x\|$ is continuous on $\left\{\Omega^{n} ; d\right\}$ and then we obtain $\|\xi\| \leqq \beta=$ $\max _{t \in I_{T}}\left\{\max _{\zeta \in G(t)}\|\zeta\|\right\}$. It is then a consequence of (6) that

$$
\left\|x_{u}\left(t^{*}\left(t_{0}, x_{0}, u\right) ; t_{0}, x_{0}\right)\right\|<\gamma+\beta
$$

and from this estimate and (5) there is obtained

$$
\left\|x_{0}\right\|^{2}+1<\left[(\gamma+\beta)^{2}+1\right] \exp 2 C T .
$$


Since $\left(t_{0}, x_{0}\right)$ may be chosen arbitrarily in $B_{\gamma}$, this completes the proof.

TheOREM 3. Assume $B_{\infty}$ is nonvoid and let $\Phi$ be a mapping of $I_{T} \times R^{n}$ into $\left\{\Omega^{m} ; d\right\}$ for which the following condition is satisfied: a bounded function $u$ on $I_{T}$ to $R^{m}$ having Lebesgue measurable components is in $U$ if and only if $u(t) \in \Phi\left(t, x_{u}\left(t ; t_{0}, x_{0}\right)\right)$ for $t \in I_{T}$; then for each $(t, x) \in B_{\infty}$, $K(t, x)$ is nonvoid.

Proof. Let us partially order $U\left(t_{0}, x_{0}\right)$ by writing $u_{1} \prec u_{2}$ if and only if $t^{*}\left(t_{0}, x_{0}, u_{1}\right) \leqq t^{*}\left(t_{0}, x_{0}, u_{2}\right)$ and $u_{1}(t)=u_{2}(t)$ almost everywhere on $\left[t_{0}, t^{*}\left(t_{0}, x_{0}, u_{1}\right)\right)$. By the Hausdorff maximality principle, the partially ordered system $\left\{U\left(t_{0}, x_{0}\right), \prec\right\}$ contains a maximal totally ordered subsystem which we denote by $V$. Defining

$$
\sigma=\sup _{\nabla} t^{*}\left(t_{0}, x_{0}, u\right)
$$

we see that there exists a nondecreasing sequence $\left\{u_{n}\right\}, u_{n} \in V$, such that $\lim _{n \rightarrow \infty} t^{*}\left(t_{0}, x_{0}, u_{n}\right)=\sigma$. Defining two elements $u_{1}, u_{2} \in\left\{U\left(t_{0}, x_{0}\right)\right.$, $\prec\}$ to be equal when both $u_{1} \prec u_{2}$ and $u_{2} \prec u_{1}$ hold, we suppose $\left\{u_{n}\right\}$ to possess infinitely many distinct elements (the finite case subsequently having an obvious treatment). Then the sequence $\left\{u_{n}\right\}$, considered as a subset of $U\left(t_{0}, x_{0}\right)$, converges in measure on $\left[t_{0}, \sigma\right)$; by virtue of 14 , Theorem $30.5 \mathrm{~s}$, p. 181], the limit, $u$, of this sequence is in $U .{ }^{4}$ As a consequence of the definition of $\left\{u_{n}\right\}, t^{*}\left(t_{0}, x_{0}, u\right)=\sigma$ so that $u \in U\left(t_{0}, x_{0}\right)$. If $\sigma=T$, we have immediately $u \in K\left(t_{0}, x_{0}\right)$ since $(T, x)$ $\notin B_{\infty}$ for all $x \in R^{n}$. If $\sigma<T$ we reach the same conclusion, for otherwise we may choose an arbitrary control $w \in U\left(\sigma, x_{u}\left(\sigma ; t_{0}, x_{0}\right)\right)$ and define a control $\bar{u} \in U\left(t_{0}, x_{0}\right)$ by

$$
\begin{array}{ll}
\bar{u}(t)=u(t), & t \in[0, \sigma), \\
\bar{u}(t)=w(t), & t \in[\sigma, T] .
\end{array}
$$

Now $\bar{u}$ may be used to extend $V$ as a totally ordered subsystem, thereby contradicting the maximality of $V$.

III. We now turn our attention to the problem of existence of optimal controls for the minimum miss distance problem. Our results are contained in

TheOREM 4. Assume $B_{\infty}$ is nonempty and let $\Phi$ be a mapping of $I_{T} \times R^{n}$ into $\left\{\Omega^{m} ; d\right\}$ satisfying the condition of Theorem 3 as well as the following conditions: (i) $\Phi$ is upper semicontinuous with respect to set inclusion; (ii) for each $(t, x) \in I_{T} \times R^{n}$ the set $R(t, x)=\{f(t, x, \phi)$ $\mid \phi \in \Phi(t, x)\}$ is convex; (iii) for each $(t, x) \in I_{T} \times R^{n}$ and each $\phi \in \Phi(t, x)$

$$
x \cdot f(t, x, \phi) \leqq C\left(\|x\|^{2}+1\right),
$$

4 It is clear that the definition of $u(t)$ on $\left[0, t_{0}\right)$ and $[\sigma, T]$ is not critical. 
where $C$ is a positive constant. Then for each $\left(t_{0}, x_{0}\right) \in B_{\infty}$ there exists a control $u^{0} \in U\left(t_{0}, x_{0}\right)$ which is optimal for the minimum miss distance problem; i.e.,

$$
\delta\left(t_{0}, x_{0}, u^{0}\right)=\min _{U\left(t_{0}, x_{0}\right)} \delta\left(t_{0}, x_{0}, u\right) .
$$

Moreover, in the set $U^{0}\left(t_{0}, x_{0}\right)$ of such optimal controls there is one, $u^{00}$, which is optimal for the minimum miss-minimum time problem; i.e.,

$$
t^{*}\left(t_{0}, x_{0}, u^{00}\right)=\min _{U_{0}\left(t_{0}, x_{0}\right)} t^{*}\left(t_{0}, x_{0}, u^{0}\right) .
$$

Proof. Except for details which we examine, our proof coincides with that of Filippov for the time optimal control problem. ${ }^{5}$ For fixed $\left(t_{0}, x_{0}\right) \in B_{\infty}$, set

$$
\lambda=\inf _{U\left(t_{0}, x_{0}\right)} \delta\left(t_{0}, x_{0}, u\right) .
$$

If $U\left(t_{0}, x_{0}\right)$ is finite, the proof is trivial. Otherwise there is a sequence $\left\{u_{n}\right\}, u_{n} \in U\left(t_{0}, x_{0}\right)$, for which $\lim _{n \rightarrow \infty} \delta\left(t_{0}, x_{0}, u_{n}\right)=\lambda$. The argument of Filippov contained in the proof of [2, Theorem 1] shows that the sequence $\left\{x_{n}\right\}, x_{n}(t) \equiv x_{u_{n}}\left(t ; t_{0}, x_{0}\right)$, is uniformly bounded and equicontinuous on $\left[t_{0}, T\right]$. There is thus a subsequence of $\left\{x_{n}\right\}$ which converges uniformly on this interval to an absolutely continuous function whose value we denote by $x(t)$. Filippov's argument [2, loc. cit.] then shows that there is a function $u^{0} \in U$ for which $x$ is a solution of (1) with $u$ replaced by $u^{0}$. In a manner similar to that of the proof of Lemma 1 we find

$$
\begin{aligned}
& \min _{\tau \in\left[t_{0}, T\right]}\left[\partial\left(x_{n}(\tau), G(\tau)\right)-\partial(x(\tau), G(\tau))\right] \\
& \leqq \delta\left(t_{0}, x_{0}, u_{n}\right)-\delta\left(t_{0}, x_{0}, u^{0}\right) \\
& \leqq \max _{\tau \in\left[t_{0}, \tau\right]}\left[\partial\left(x_{n}(\tau), G(\tau)\right)-\partial(x(\tau), G(\tau))\right]
\end{aligned}
$$

From this estimate, together with the continuity of $\partial, G$ and the uniform convergence of a subsequence of $\left\{x_{n}\right\}$ to $x$, we conclude that $\delta\left(t_{0}, x_{0}, u^{0}\right)=\lambda$. Hence $u^{0} \in U\left(t_{0}, x_{0}\right)$ and the proof of the first assertion is complete.

Now let us set

$$
\eta=\inf _{U^{0}\left(t_{0}, x_{0}\right)} t^{*}\left(t_{0}, x_{0}, u^{0}\right)
$$

- A proof can be given which displays Theorem 4 as a corollary of Filippov's theorem; however, we believe the present proof casts more light on the relationship between Theorem 4 and the preceding results. 
certainly $\eta \geqq t_{0}$ and we wish to show first of all that $\eta>t_{0}$. If $U^{0}\left(t_{0}, x_{0}\right)$ is finite this demonstration, as well as the proof of the second assertion of the theorem, is trivial. Hence, assuming $U^{0}\left(t_{0}, x_{0}\right)$ to be infinite, there is a sequence $\left\{v_{n}\right\}, v_{n} \in U^{0}\left(t_{0}, x_{0}\right)$, such that $\lim _{n \rightarrow \infty} t^{*}\left(t_{0}, x_{0}, v_{n}\right)=\eta$. Since $U^{0}\left(t_{0}, x_{0}\right) \subset K\left(t_{0}, x_{0}\right)$ we see that if $\eta=t_{0}$ then every neighborhood of $\left(t_{0}, x_{0}\right)$ intersects the complement of $B_{\infty}$. This contradicts Theorem 1 , so we conclude that $\eta>t_{0}$. Since the sequence $\left\{y_{n}\right\}$, $y_{n}(t) \equiv x_{v_{n}}\left(t ; t_{0}, x_{0}\right)$, is uniformly bounded and equicontinuous on $\left[t_{0}, T\right]$ we may conclude as above that there exists $v^{0} \in U$ for which the solution of (1), with $u$ replaced by $v^{0}$, is the uniform limit on $\left[t_{0}, T\right]$ of a subsequence of $\left\{y_{n}\right\}$. By virtue of $(7)$ we see that $v^{0} \in U^{0}\left(t_{0}, x_{0}\right)$ and thus $\eta \leqq t^{*}\left(t_{0}, x_{0}, v^{0}\right)$. On the other hand, denoting by $y(t)$ the value of the solution of (1) corresponding to $v^{0}$ and taking a subsequence of $\left\{y_{n}\right\}$ if necessary, we find by virtue of the continuity of the functions involved that

$$
\begin{aligned}
\lim _{n \rightarrow \infty} \delta\left(t_{0}, x_{0}, v_{n}\right) \\
\quad=\lim _{n \rightarrow \infty} \partial\left(y_{n}\left(t^{*}\left(t_{0}, x_{0}, v_{n}\right)\right), G\left(t^{*}\left(t_{0}, x_{0}, v_{n}\right)\right)\right)=\partial(y(\eta), G(\eta)) .
\end{aligned}
$$

Since $\delta\left(t_{0}, x_{0}, v_{n}\right)$ is independent of $n$, (8) implies that $\eta \geqq t^{*}\left(t_{0}, x_{0}, v_{0}\right)$ and this together with the above result yields $\eta=t^{*}\left(t_{0}, x_{0}, v^{0}\right)$. With the identification $u^{00}=v^{0}$ the proof of the theorem is complete.

Finally let us consider the set $B$ defined by

$$
B=\left\{(t, x) \in B_{\infty} \mid \delta(t, x, u)=0 \text { for some } u \in U(t, x)\right\} .
$$

Evidently if $Q$ is not empty then $B_{0}$ is not empty; conversely we have

COROLlaRY 1. Under the conditions of Theorem 4 , if $B_{0}$ is not empty then $B$ is not empty and therefore $B=B_{0}$.

Proof. As a consequence of Theorem 4 and the definition of $B_{\gamma}$, if $\left(t_{0}, x_{0}\right) \in B_{0}$ then there is a sequence $\left\{w_{n}\right\}, w_{n} \in U^{0}\left(t_{0}, x_{0}\right)$, such that $\delta\left(t_{0}, x_{0}, w_{n}\right)<n^{-1}$. An argument like that of Theorem 4 permits us to conclude that there exists $\bar{u} \in U^{0}\left(t_{0}, x_{0}\right)$ such that $\delta\left(t_{0}, x_{0}, \bar{u}\right)=0$.

By virtue of Corollary 1 and Theorem 1, the set $B$ is a Borel set when the conditions of Theorem 4 are satisfied. Hence, denoting by $\mu$ Lebesgue measure in $I_{T} \times R^{n}, \beta$ is $\mu$-measurable. Moreover, if the condition (iii) of Theorem 4 is strengthened by replacing $x \cdot f$ by $|x \cdot f|$, then it follows from Theorem 2 that $\mu(B)<\infty$. The following open question is of interest: what conditions suffice to ensure that $\mu(B)>0$ ?

IV. Throughout this section we assume that $G(t)=\{0\}$ for $t \in I_{T}$ and that $\Phi$ is a mapping of $I_{T} \times R^{n}$ into $\left\{\Omega^{m} ; d\right\}$ having a constant 
value $\bar{\Phi}$ and satisfying the condition of Theorem 3 . We define

$$
\begin{aligned}
\Psi(t, x) & =\{\phi \in \bar{\Phi} \mid x \cdot f(t, x, \phi)<0\} ; \\
D & =\left\{(t, x) \in I_{T} \times R^{n} \mid \Psi(t, x) \text { is nonvoid }\right\} .
\end{aligned}
$$

TheOREM 5. $B_{\infty}$ is nonvoid if and only if $D$ is nonvoid; moreover, $a$ point $\left(t_{0}, x_{0}\right) \in B_{\infty}$ only if $D$ has a nonvoid intersection with the set of points attainable from $\left(t_{0}, x_{0}\right)$ with controls in $U$.

Proof. If $\left(t_{0}, x_{0}\right) \in D$ then there exists $\phi_{0} \in \Psi\left(t_{0}, x_{0}\right)$; by continuity of $f$ there exists $t_{2} \in\left(t_{0}, T\right]$ such that

$$
x_{\bar{u}}\left(t ; t_{0}, x_{0}\right) \cdot f\left(t, x_{\bar{u}}\left(t ; t_{0}, x_{0}\right), \phi_{0}\right)<0, t \in\left[t_{0}, t_{1}\right),
$$

where $\bar{u}(t) \equiv \phi_{0}$ on $\left[t_{0}, T\right]$. From

$$
\begin{aligned}
\left\|x_{0}\right\|^{2}- & \left\|x_{u}\left(t ; t_{0}, x_{0}\right)\right\|^{2} \\
& =-2 \int_{t_{0}}^{t} x_{u}\left(\lambda ; t_{0}, x_{0}\right) \cdot f\left(\lambda, x_{u}\left(\lambda ; t_{0}, x_{0}\right), u(\lambda)\right) d \lambda
\end{aligned}
$$

there follows then $\left\|x_{0}\right\|^{2}>\left\|x_{u}\left(t_{1} ; t_{0}, x_{0}\right)\right\|^{2}$ so that $D \subset B_{\infty}$.

Conversely, let $\left(t_{0}, x_{0}\right) \in B_{\infty}$; then for $u \in U\left(t_{0}, x_{0}\right)$,

$$
\begin{aligned}
0 & <\left\|x_{0}\right\|^{2}-\left\|x_{u}\left(t^{*}\left(t_{0}, x_{0}, u\right) ; t_{0}, x_{0}\right)\right\|^{2} \\
& =-2 \int_{t_{0}}^{t^{*}\left(t_{0}, x_{0}, u\right)} x_{u}\left(\lambda ; t_{0}, x_{0}\right) \cdot f\left(\lambda, x_{u}\left(\lambda ; t_{0}, x_{0}\right), u(\lambda)\right) d \lambda .
\end{aligned}
$$

From (10) a contradiction is obtained if it be assumed that $x \cdot f(t, x, \phi)$ $\geqq 0$ on $I_{T} \times R^{n} \times \bar{\Phi}$; hence, $D$ is nonvoid.

For the final assertion, we note that if $\left(t_{0}, x_{0}\right) \in B_{\infty}$, the assumption that $\left(t, x_{u}\left(t ; t_{0}, x_{0}\right)\right) \notin D$ on $\left[t_{0}, t^{*}\left(t_{0}, x_{0}, u\right)\right)$ for every $u \in U\left(t_{0}, x_{0}\right)$ leads to a contradiction in the same way as that obtained from (10).

ADDED IN PROOF. Depending as it does on Theorem 1, the proof we have given for the final assertion of Theorem 4 is not valid without an additional restriction, such as that imposed in $\S \mathrm{IV}$, on $\Phi$. A similar comment applies to the final paragraph of $\S I I I$.

\section{REFERENCES}

1. T. F. Bridgland, Jr., On the existence of optimal feedback controls, J. SIAM Ser. A Control, 1 (1963), 261-274.

2. A. F. Filippov, On certain questions in the theory of optimal control (transl. by L. W. Neustadt), J. SIAM Ser. A Control 1 (1962), 76-84.

3. E. A. Coddington and N. Levinson, Theory of ordinary differential equations, McGraw-Hill, New York, 1955.

4. E. J. McShane, Integration, Princeton Univ. Press, Princeton, N. J., 1947.

University of Alabama Research Institute, Huntsville and University of South Carolina 